\title{
Niclosamide for Covid-19: bridging the gap
}

\author{
Hayder M. Al-kuraishy ${ }^{1} \cdot$ Ali I. Al-Gareeb $^{1} \cdot$ Khalid J. Alzahrani $^{2} \cdot$ Athanasios Alexiou $^{3,4} \oplus$ - Gaber El-Saber Batiha ${ }^{5}$
}

Received: 10 June 2021 / Accepted: 17 August 2021 / Published online: 18 October 2021

(c) The Author(s), under exclusive licence to Springer Nature B.V. 2021

\begin{abstract}
Aim/purpose Niclosamide (NCL) is an anthelminthic drug, which is widely used to treat various diseases due to its pleiotropic anti-inflammatory and antiviral activities. NCL modulates of uncoupling oxidative phosphorylation and different signaling pathways in human biological processes. The wide-spectrum antiviral effect of NCL makes it a possible candidate for recent pandemic SARS-CoV-2 infection and may reduce Covid-19 severity. Therefore, the aim of the present study was to review and clarify the potential role of NCL in Covid-19.

Methods This study reviewed and highlighted the protective role of NCL therapy in Covid-19. A related literature search in PubMed, Scopus, Web of Science, Google Scholar, and Science Direct was done.

Results NCL has noteworthy anti-inflammatory and antiviral effects. The primary antiviral mechanism of NCL is through neutralization of endosomal PH and inhibition of viral protein maturation. NCL acts as a proton carrier, inhibits homeostasis of endosomal PH, which limiting of viral proliferation and release. The anti-inflammatory effects of NCL are mediated by suppression of inflammatory signaling pathways and release of pro-inflammatory cytokines. However, the major limitation in using NCL is low aqueous solubility, which reduces oral bioavailability and therapeutic serum concentration that reducing the in vivo effect of NCL against SARS-CoV-2.

Conclusions NCL has anti-inflammatory and immune regulatory effects by modulating the release of pro-inflammatory cytokines, inhibition of NF- $\mathrm{BB} / \mathrm{NLRP} 3$ inflammasome and mTOR signaling pathway. NCL has an anti-SARS-CoV-2 effect via interruption of viral life-cycle and/or induction of cytopathic effect. Prospective clinical studies and clinical trials are mandatory to confirm the potential role of NCL in patients with Covid-19 concerning the severity and clinical outcomes.
\end{abstract}

Keywords Covid-19 $\cdot$ SARS-CoV-2 infection $\cdot$ Niclosamide $\cdot$ Acute respiratory distress syndrome $\cdot$ Angiotensin-converting enzyme

\section{Introduction}

Athanasios Alexiou

alextha@yahoo.gr

$\triangle$ Gaber El-Saber Batiha

alextha@yahoo.gr

1 Department of Clinical Pharmacology and Medicine, College of Medicine, ALmustansiriyia University, Baghdad, Iraq

2 Department of Clinical Laboratories Sciences, College of Applied Medical Sciences, Taif University, PO Box 11099, Taif 21944, Saudi Arabia

3 Department of Science and Engineering, Novel Global Community Educational Foundation, Hebersham, Australia

4 AFNP Med Austria, Wien, Austria

5 Department of Pharmacology and Therapeutics, Faculty of Veterinary Medicine, Damanhour University, Damanhour 22511, El Beheira, Egypt
Coronavirus disease 2019 (Covid-19) is a worldwide pandemic disease caused by severe acute respiratory coronavirus 2 (SARS-CoV-2). Covid-19 affects diverse organs, chiefly the respiratory system, and presented with acute lung injury (ALI) and acute respiratory distress syndrome (ARDS) [1]. The systemic effect of Covid-19 is due to the broad allocation and distribution of angiotensin-converting enzyme 2 (AEC2), a primary receptor for SARS-CoV-2 [2, 3]. ACE2 receptor is primarily expressed in lung alveolar cells type II, proximal renal tubules, immune cells, and intestines [4]. Binding of SARS-CoV-2 with ACE2 is linked with downregulation of these protective receptors with significant intensification in the level of vasoconstrictors angiotensin II (Ang II) and decreasing of vasodilator angiotensin (Ang 1-7), (Ang 1-9) with induction release of pro-inflammatory 
cytokines. These changes elicit hyper-inflammation and the development of cytokine storms that linked with ARDS development and multi-organ failure (MOF) [5].

Since the World Health Organization (WHO) declaration of this disease as a pandemic and until late July 2021, the total confirmed cases are $194,250,977$, with 4,258,789 deaths. In this international predicament, extraordinary efforts, advancing investigations, and research are meticulously performed to find an effective drug against SARSCoV-2 from recent or old standard and/or approved drugs as repurposing drug strategy [6].

Niclosamide (NCL) is an anthelminthic drug, inhibits glucose uptake by the worms, used to treat tapeworm infestations, including taeniasis, hymenolepiasis, and diphyllobothriasis. However, it was not effective against other types of worms [7]. NLS was discovered in 1953 in Bayer Research Laboratories as a molluscicidal agent against schistosomiasis snail and was marketed as Bayluscide in 1959 [8]. Later on, it was to be effective for tapeworms in 1962 and marketed as Yomesan for human use; in 1982, it was approved by Food and Drug Administration (FDA) for treatment of tapeworms and regarded as one of essential medicine by World Health Organization (WHO) [9]. NCL is widely used to treat various metabolic disorders, cancers, viral infections, and immunological disturbance due to its pleiotropic effects antiviral and anti-inflammatory properties with modulation of uncoupling oxidative phosphorylation and various signaling pathways in human biological processes [10]. Several experimental studies confirmed that NCL is a safe and welltolerated drug with a toxicity profile of more than $5 \mathrm{~g} / \mathrm{kg}$ in rats [11]. NCL is well tolerated drug in humans, can be used orally at $2 \mathrm{~g} /$ day for worm treatment leading to serum concentrations of $0.25-6.0 \mu \mathrm{g} / \mathrm{mL}$ that corresponding to $0.76-18.35 \mu \mathrm{M}$ respectively. This concentration is not toxic and within range of active antiviral concentration in humans [8]. Since, NCL has potential antiviral and anti-inflammatory properties, therefore, the aim of the present study was to review and clarify the potential role of NCL in Covid-19.

\section{Methods and search strategy}

An interrelated literature search in PubMed, Scopus, Web of Science, Google Scholar, and Science Direct was prepared. All of published articles related to the role of NCL and Covid-19 were included in this mini-review. We seek out the international database by using the medical subject heading $(\mathrm{MeSH})$ to recognize the applicable articles published up to 2021. The listed keywords used in this search included; [Covid-19 OR SARS-CoV-2] AND [NCL], [Covid-19 OR SARS-CoV-2] AND [NCL], [antiviral, antiinflammatory effects], AND [Covid-19 severity]. The entire types of published articles with dissimilar languages were integrated, and the absolute findings were sum up in a minireview study.

\section{Antiviral activity of Niclosamide}

The antiviral activity of NCL against influenza virus and human rhinovirus was explained to be $\mathrm{PH}$-dependent and related to the protonophore/proton carrier activity. NCL has neutralizing effects on the endo-lysosomal $\mathrm{PH}$ with significant acidification of extracellular medium [12]. NCL did not affect cell H-ATPase but neutralize coated vesicles indicating a proton carrier inhibition independent of any target proteins [12].Wang et al. [13] illustrated that NCL inhibits entry and transmission of the Chikungunya virus, which enters the host cells through receptor-mediated endocytosis, suggesting that NCL might be effective in treatment of Chikungunya virus and other alphaviruses. Similarly, NCL inhibits replication of Zika virus and attenuates Zika virusinduced cell death through attenuation of $\mathrm{PH}$-dependent membrane fusion [14]. Indeed, NCL suppresses viral-lytic replication of Epstein-Barr virus (EBV) through inhibition of the mammalian cellular target of rapamycin (mTOR) activation, which is necessary for pathogenesis of EBV infection [15]. Recently, Kao et al. [16] observed that NCL has a potential effect against Dengue infection by inhibiting various viral life-cycle and virulence steps, including binding, receptor-mediated endocytosis entry, fusion, uncoating, replication, assembly, and RNA release. In addition, NCL inhibits Dengue and Zika infections through targeting of NS2B-NS3 protease [66]. Furthermore, more extensive experimental studies illustrated that NCL has more extended antiviral spectrums. NLS inhibits replication of hepatitis C virus $(\mathrm{HCV})$ through modulation of host immune response [17] and regarded as a potent inhibitor of Ebola virus [18]. As well, NLS reduces replication of human adenovirus by blocking of viral protein-endosomal transport [19]. Of note, most of in vitro studies revealed short-term antiviral effects of NLS; however long-term effect was confirmed against HIV infection through inhibition replication of human HIV in the macrophage [67].

This wide-spectrum antiviral effect of NCL makes it possible for recent pandemic SARS-CoV-2 infection and may reduce Covid-19 severity.

\section{Coronaviruses and niclosamide}

Coronaviruses (CoVs) are a group of non-segmental enveloped, positive-sense RNA viruses with a sizeable genomic size of about 27-34 kb [20]. Human strain of CoVs like HCoV-229E, HCoV-HKU1, HCoV-OC43, and HCoV-NL63 may cause self-limiting and mild upper respiratory tract infection [21]. In July 2003, the SARS-CoV outbreak was 
emerged and led to pandemics in China and associated with a 9\% mortality rate [22]. In 2012, Middle East Respiratory Syndrome (MERS) emerged in Saudi Arabia, which was caused by MERS-CoV led to more fatality with a $34 \%$ mortality rate [23]. At the time of the SARS-CoV outbreak, there was no specific effective against replication of Coronaviruses (CoVs). NCL has been reported to be effective against SARS by inhibiting SARS-CoV replication with protection from viral cytopathic effects at $1.56 \mu \mathrm{M}$ concentration. The anti-cytopathic effect of NCL was demonstrated at a lower concentration of $1.0 \mu \mathrm{M}$ in Vero E6 cells [24]. The effective concentration of NCL that reduces $50 \%$ of cell viability of infected cells was 1-3 $\mu \mathrm{M}$. As well, NCL was able to inhibit replication of SARS-CoV when the cells infected, suggesting that NCL does not interferes with SARS-CoV attachment and entry to the affected cells [24]. Besides, $5 \mu \mathrm{M}$ leads to more potent inhibition of SARS-CoV as compared to 1-3 $\mu \mathrm{M}$, indicating a dose-dependent anti-SARS-CoV activity of NCL [24, 68].

Various molecular studies regarding SARS-CoV genomic analysis confirmed that SARS-CoV 3CL-protease is an important enzyme for processing viral polyprotein and is regarded as a potential drug target [69]. However, NCL showed no noticeable effect on SARS-CoV 3CL-protease even at a higher concentration [25]. Furthermore, NCL inhibits MERS-CoV replication by inhibiting E3-ligase S-phase kinase associated protein 2 (SKP2), which is involved in the degradation of proteasomes $[26,70]$.

\section{Covid-19 and niclosamide}

It has been shown that SARS-CoV-2 has a genomic similarity with SARS-CoV in about $79 \%$ and with that of bat $\mathrm{CoV}$ in about $96 \%$ [27]. Therefore, experimental studies that confirmed the potential drug effects on SARS-CoV could be applied for SARS-CoV-2 due to relative genomic similarity. Molecular docking and in vitro studies illustrated that NCL is an effective agent against SARS-CoV-2 replication by inhibiting protease enzyme [28, 29]. Different recent studies illustrated that NCL inhibits SARS-CoV-2 replication through inhibition of intracellular acidification and viral protein assembly [30] with autophagy activation [63] thereby; it can be used in the management of Covid-19.

The major limitation in using of NCL is low aqueous solubility, which reduces oral bioavailability and therapeutic serum concentration that reduces in vivo effect of NCL against SARS-CoV-2[31]. Therefore, different pharmaceutical formulations and/or routes are applied for NCL to be more effective in the management of SARS-CoV-2 infections and to overcome the low bioavailability of NCL. Brunaugh et al. [32] revealed that inhalation of NCL in combination with human lysosome increases in vitro and vivo activity of NCL against SARS-CoV-2 that ensures a rapid clinical improvement. Besides, Wang et al. [33] confirmed that lipid nanoparticles of NCL are cost-effective and highly scalable against SARS-CoV-2 in Vero E6 and AEC2 expressing cells. Jara et al. [34] experimental study involved multi-dose pharmacokinetics illustrated that inhalational route of NCL is more effective against pulmonary SARS$\mathrm{CoV}-2$ infection in the Syrian hamster model. Therefore, mitigation of NCL through these pharmaceutical formulations may improve its solubility and pharmacokinetic profile.

The main antiviral mechanisms of NCL are neutralization of endosomal $\mathrm{PH}$ and inhibition of viral protein maturation in the Golgi apparatus, chiefly in CoVs and hepatitis $\mathrm{C}$ virus (HCV). [12] NCL acts as a proton carrier and inhibits homeostasis of endosomal $\mathrm{PH}$, limiting viral proliferation, and release [12]. Therefore, the antiviral mechanisms of NCL are either of PH-dependent, which inhibit endosomal acidification, or PH-independent pathway. NCL inhibits mitochondrial oxidative phosphorylation with subsequent depletion of intracellular ATP, which trigger adenosine monophosphate protein kinase (AMPK) which directly blocks mTOR signaling pathway [76, 77] (Fig. 1).

On the other hand, NCL has anti-inflammatory and immune regulatory effects that may affect the propagation of different inflammatory and immune-mediated disorders through inhibition of pro-inflammatory cytokine, mainly tumor necrosis factor-alpha (TNF-a) [35]. Moreover, experimental studies demonstrated that NCL is a potent inhibitor of $\mathrm{Ca}^{+2}$ - activated $\mathrm{Cl}^{-}$channel TMEM16A expressed in the airway smooth muscles and mucus-producing goblet cells that mediates bronchoconstriction and mucus hypersecretion in chronic inflammatory airway diseases[36, 64]. Miner et al., revealed that NCL had beneficial effect on constricted airways and mucus hyper-secretion during airway inflammation by antagonizing of airway TMEM16A channels [65].

In addition, NCL inhibits progression of inflammatory airway diseases like asthma, chronic obstructive pulmonary disease, and cystic fibrosis by suppression release of IL-8 and intracellular $\mathrm{ca}^{+2}$ signaling [36].

What's more, NCL is effective in treating pulmonary secondary bacterial infections caused by pseudomonas species [37]. Of note, NCL might be an effective agent against Covid-19 pneumonia through anti-SARS-CoV-2 activity and modulation of associated inflammatory disorders [38]. NCL improves anti-inflammatory and inhibits pro-inflammatory cytokines, limiting the development of cytokine storminduced ALI in Covid-19 [39]. Therefore, NCL may reduce the severity of Covid-19 by attenuating the development of ALI and ARDS induced by SARS-CoV-2 infection [40]. Braga et al. [41] showed that NCL reduces the development of pulmonary hypertension $(\mathrm{PH})$ in rats through inhibition of signal transducer and activator transcription 3 (STAT-3). It has been reported that $\mathrm{PH}$ is developed during SARS-CoV-2 infection and associated with Covid-19 severity and high 


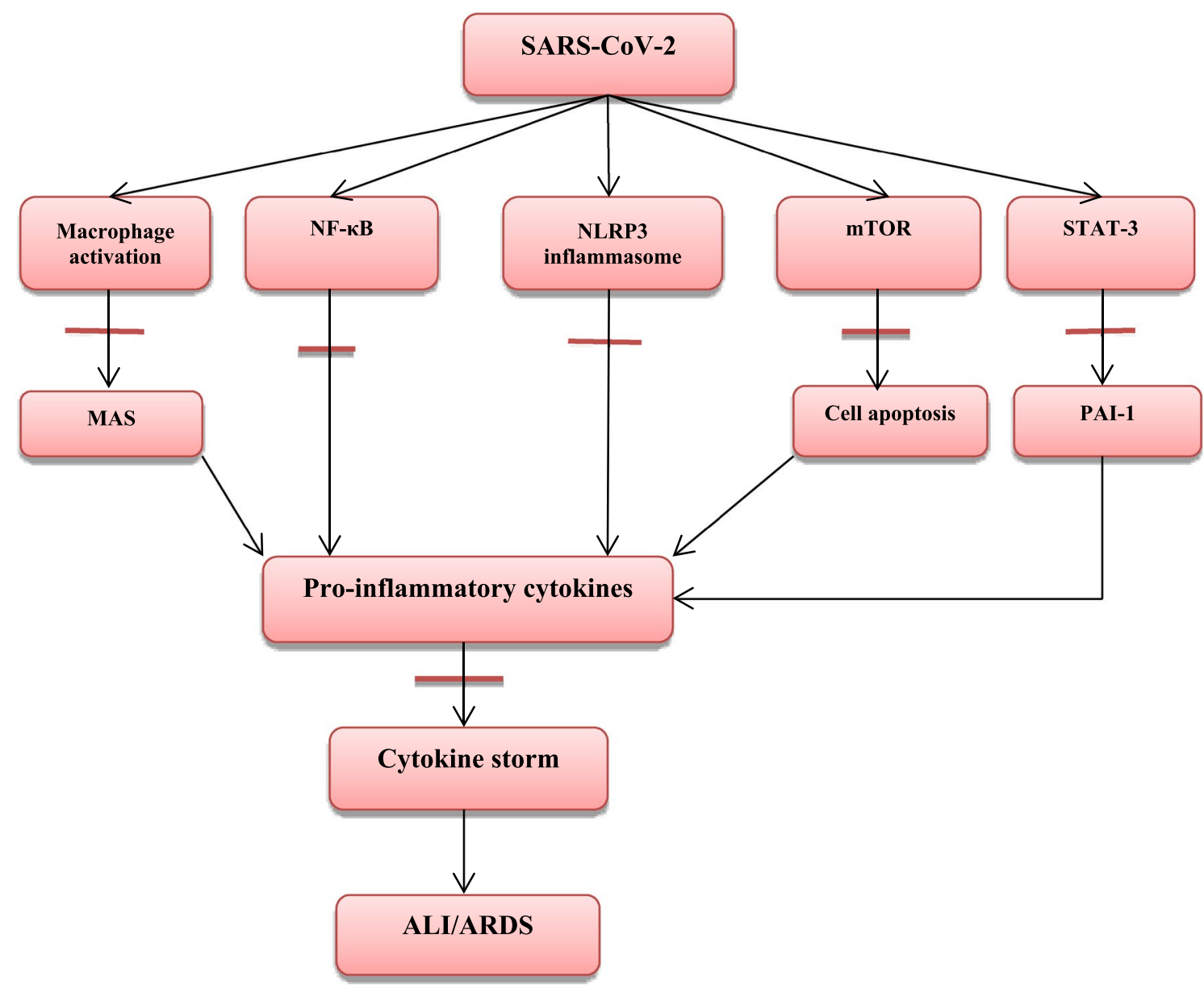

Fig. 1 Inhibitory effects of niclosamide on SARS-CoV-2 infection and associated inflammatory pathways: SARS-CoV-2 through activation of macrophage, mammalian target of rapamycin (mTOR) and signal transducer and activator transcription 3 (STAT-3) leads to macrophage activation syndrome (MAS), cell apoptosis, and activation release of plasminogen activator inhibitor-1(PAI-1) respectively. As well, SARS-CoV-2 directly activates nuclear factor kappa B (NF-

mortality [42]. Recent studies showed that SARS-CoV-2 proteins, mainly ORF6 induce dysfunction of STAT-3 with compensatory over-activation of STAT-3-induced activation of plasminogen activator inhibitor-1(PAI-1) and subsequent coagulopathy and thrombosis [43]. In this way, overactivated PAI-1 stimulates macrophages to produce pro-inflammatory cytokines, leading to more inhibition of STAT-3 in a vicious cycle [44].

High circulating IL-6 level in patients with Covid-19 is implicated in the development of cytokine storm and production of PAI-1, so inhibition of IL-6 by tocilizumab may alleviate clinical outcomes through reduction synthesis of PAI-1 in severely affected Covid-19 patients [45]. For that reason, inhibition of STAT-3, PAI-1, and IL-6 is related with better clinical outcomes in Covid-19 patients, and the use of NCL might be beneficial in this context. Different studies
кB) and nod-like receptor pyrin 3 (NLRP3) inflammasome leading to release of pro-inflammatory cytokines with development of acute lung injury (ALI) and acute respiratory distress syndrome (ARDS). Niclosamide has inhibitory effects ( 2-mediated inflammatory signaling pathways and release of proinflammatory cytokines

reported that NCL attenuates the production of STAT-3, PAI-1, and IL-6 through the inhibition of associated inflammatory pathways during cancer metastasis [46, 47]. At present, there are no clinical studies accounted for the potential effects of NCL on the inflammatory cytokines and pathway in Covid-19 patients. In SARS-CoV-2, the mammalian target of rapamycin (mTOR) is activated with inhibition of p53. Activation of mTOR inhibits host cells for interferon (INF) production, while inhibition of p53 is associated with high viral survival, so mTOR inhibitors and p53 activators might attenuate SARS-CoV-2 [48]. Gassen et al. [49] found that NCL activates autophagy and inhibits the mTOR pathway in SARS-CoV-2, which might explain the prolonged antiviral effect of NCL. NCL plays a crucial role in the activation of p53 in small cell lung cancer [50] and viral infections [51]; therefore, it may attenuate the survival of SARS-CoV-2. Of 
interest, most of enveloped RNA viruses including SARSCoV-2 and HIV-1 activate mTOR pathway during their replications, thus disruption of this pathway may interfere with viral replication and pathogenesis [72].

Also, stimulation of nod-like receptor pyrin 3 (NLRP3) inflammasome and nuclear factor kappa B (NF- $\kappa \mathrm{B})$ signaling pathway are associated with SARS-CoV-2 infection, leading to the release of pro-inflammatory cytokines, which initiate cytokine storm with development of ALI and ARDS [52, 53]. Recently, Hu et al. [54] observed that chemical mitochondrial uncouplers like NCL have a potential inhibitory effect on the NLRP3 inflammasome via suppressing NF- $\kappa B$ signaling pathway. Thus, NCL has an anti-inflammatory effect that may mitigate hyperinflammatory status and hypercytokinemia during acute SARS-CoV-2 infection and may reduce risk of ARDS [55].

Despite of these beneficial effects of NCL, it may increase of oxidative stress by induction of mitochondrial dysfunction [56]. At this point, NCL may aggravate SARS-CoV-2 infection-induced oxidative stress and may be linked with Covid-19 severity [57]. However, Chen et al. [71] illustrated that NCL-induced oxidative stress could be beneficial in the management of cervical cancer through inhibition of mTOR signaling pathway.

Of interest, male subjects are highly susceptible to the SARS-CoV-2 infection than females due to high androgen level, which enhances SARS-CoV-2 entry through activation of transmembrane protease serine 2 (TMPRSS2) [58]. Liu et al. [59] confirmed that NCL blocks androgen receptors in prostatic carcinoma. Therefore, depending on this evidence, NCL may produce more protection against SARS-CoV-2 infection in men compared to women.

On the other hand, NCL has immune regulatory and modulatory effects on the immune response by inhibiting dendritic cells, $T$ cell proliferation, and antigen response with inhibition of NF- $\kappa \mathrm{B} / \mathrm{NLRP} 3$ inflammasome and mTOR signaling pathway [60]. Experimental studies illustrated that NCL has a dose-dependent effect in inhibiting macrophage function and secretion of pro-inflammatory cytokines via suppression of SATA-3/ NF- $\kappa$ B signaling pathway [61]. It has been shown by Ruscitti et al. [62] that macrophage activation syndrome (MAS) is linked with the development of cytokine storm, ALI, ARDS, poor clinical outcomes, and high mortality rate in severely affected Covid-19 patients [62]. Thus, it will be expected that using of NCL in Covid19 patients will produce a more significant protective effect through suppression of MAS.

It has been shown that endothelial dysfunction is associated with severe Covid-19, leading to pulmonary endothelial dysfunction and thrombotic disorders [73]. Experimental study demonstrated that NCL attenuate endothelial dysfunction through activation release of nitric oxide (NO) from vascular endothelium with stimulation of cyclic guanosine monophosphate (cAMP) [74]. Likewise, NCL reduces SARS-CoV-2-induced platelet hyper-reactivity and thrombotic disorders [75].

The net effects of NCL on SARS-CoV-2 infection and associated inflammatory and immunological derangements in Covid-19 are summarized (Fig. 2). This study highlighted the protective role of NCL therapy in Covid-19 from in vitro and docking studies; however, prospective clinical studies and clinical trials are mandatory in this regard to confirm the potential role of NCL in patients with Covid-19 concerning the severity and clinical outcomes.

\section{Conclusion}

NCL has anti-inflammatory and immune regulatory effects by modulating the release of pro-inflammatory cytokines, inhibition of NF- $\kappa \mathrm{B} / \mathrm{NLRP} 3$ inflammasome and mTOR signaling pathway. NCL has an anti-SARS-CoV-2 effect via interruption of viral life-cycle and/or induction of cytopathic effect. The present findings cannot give a conclusion regarding the ultimate effectiveness of NCL in Covid-19 patients. Prospective clinical studies and clinical trials are required to authorize the potential role of NCL in patients with Covid19 regarding the severity and clinical outcomes. 
Fig. 2 Antiviral mechanisms of niclosamide: the antiviral mechanisms of niclosamide (NCL) are either of PHdependent, which inhibit endosomal acidification, or PH-independent pathway. NCL inhibits mitochondrial oxidative phosphorylation with subsequent depletion of intracellular ATP, which trigger adenosine monophosphate protein kinase (AMPK) which directly blocks mTOR signaling pathway

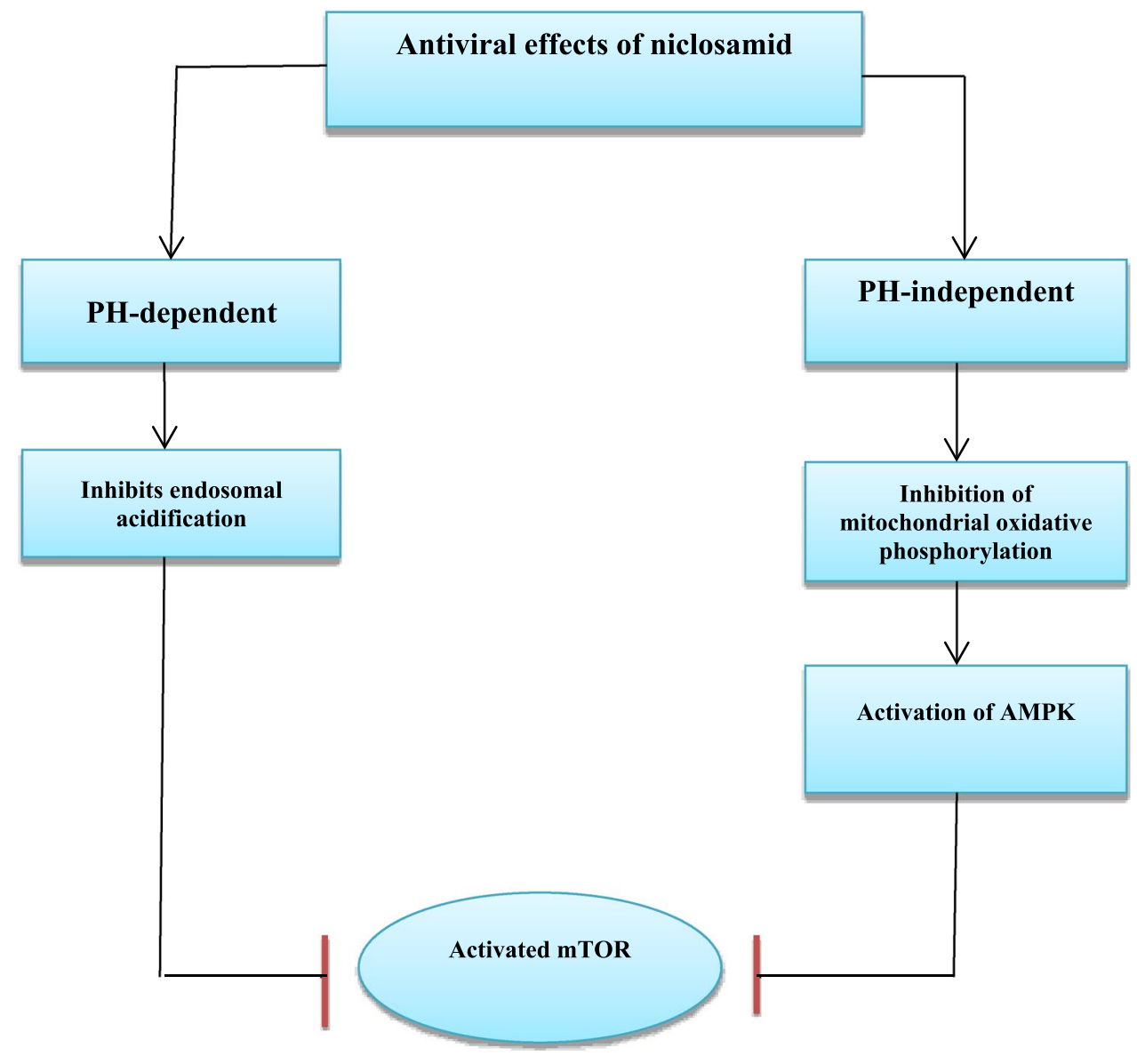

Author contributions Manuscript preparation: HMAK, AIAG, KJA. Manuscript editing: GELSB, AA.

Funding This research received no external funding.

\section{Declarations}

Conflict of interest The authors declare that they have no competing interests.

\section{References}

1. Lugnier C, Al-Kuraishy HM, Rousseau E (2021) PDE4 inhibition as a therapeutic strategy for improvement of pulmonary dysfunctions in Covid-19 and cigarette smoking. Biochem Pharmacol 185:114431

2. Al-Kuraishy HM, Al-Gareeb AI, Cruz-Martins N, Batiha GE (2021) Hyperbilirubinemia in Gilbert syndrome attenuates Covid19 induced-metabolic disturbances: a case-report study. Front Cardiovasc Med 8:71

3. Al-Kuraishy HM, Al-Gareeb AI, Al-Niemi MS, Al-Buhadily AK, Al-Harchan NA, Lugnier C (2020) COVID-19 and phosphodiesterase enzyme type 5 inhibitors. J Microsc Ultrastructure 8(4):141

4. Al-Kuraishy HM, Al-Naimi MS, Lungnier CM, Al-Gareeb AI (2020) Macrolides and COVID-19: an optimum premise. Biomed Biotechnol Res J 4(3):189
5. Al-Kuraishy HM, Hussien NR, Al-Naimi MS, Al-Buhadily AK, Al-Gareeb AI, Lungnier C (2020) Renin-Angiotensin system and fibrinolytic pathway in COVID-19: One-way skepticism. Biomed Biotechnol Res J 4(5):33

6. Galindez G, Matschinske J, Rose TD, Sadegh S, Salgado-Albarrán M, Späth J, Baumbach J, Pauling JK (2021) Lessons from the COVID-19 pandemic for advancing computational drug repurposing strategies. Nat Comput Sci 1(1):33-41

7. Al-Gareeb AI, Aljubory KD, Alkuraishy HM (2017) Niclosamide as an anti-obesity drug: an experimental study. Eating and Weight Disorders-Studies on Anorexia, Bulimia and Obesity 22(2):339-344

8. Andrews P, Thyssen J, Lorke D (1982) The biology and toxicology of molluscicides. Bayluscide Pharmacol Ther 19(2):245-295

9. World Health Organization (2009) The selection and use of essential medicines: report of the WHO Expert Committee, March 2009 (including the 16th WHO model list of essential medicines and the 2nd WHO model list of essential medicines for children). World Health Organization, Geneva

10. Chen W, Mook RA Jr, Premont RT, Wang J (2018) Niclosamide: beyond an antihelminthic drug. Cell Signal 41:89-96

11. AwAD OM (1995) Assessment of the developmental toxicity of in utero exposure of Wistar albino rats to ametryne and Niclosamide. Pestic Biochem Physiol 53(1):1-9

12. Jurgeit A, McDowell R, Moese S, Meldrum E, Schwendener R, Greber UF (2012) Niclosamide is a proton carrier and targets acidic endosomes with broad antiviral effects. PLoS Pathog 8(10):e1002976 
13. Wang YM, Lu JW, Lin CC, Chin YF, Wu TY, Lin LI, Lai ZZ, Kuo SC, Ho YJ (2016) Antiviral activities of Niclosamide and nitazoxanide against chikungunya virus entry and transmission. Antiviral Res 135:81-90

14. Xu M, Lee EM, Wen Z, Cheng Y, Huang WK, Qian X et al (2016) Identification of small-molecule inhibitors of Zika virus infection and induced neural cell death via a drug repurposing screen. Nat Med 22:1101-1107

15. Huang L, Yang M, Yuan Y, Li X, Kuang E (2017) Niclosamide inhibits lytic replication of Epstein-Barr virus by disrupting mTOR activation. Antiviral Res 138:68-78

16. Kao JC, HuangFu WC, Tsai TT, Ho MR, Jhan MK, Shen TJ, Tseng PC, Wang YT, Lin CF (2018) The antiparasitic drug niclosamide inhibits dengue virus infection by interfering with endosomal acidification independent of mTOR. PLoS Negl Trop Dis 12(8):6715

17. Korba BE, Elazar M, Lui P, Rossignol JF, Glenn JS (2008) Potential for hepatitis $\mathrm{C}$ virus resistance to nitazoxanide or tizoxanide. Antimicrob Agents Chemother 52(11):4069-4071

18. Madrid PB, Panchal RG, Warren TK, Shurtleff AC, Endsley AN, Green CE, Kolokoltsov A, Davey R, Manger ID, Gilfillan L, Bavari S (2015) Evaluation of Ebola virus inhibitors for drug repurposing. ACS Infect Dis 1(7):317-326

19. Marrugal-Lorenzo JA, Serna-Gallego A, Berastegui-Cabrera J, Pachón J, Sánchez-Céspedes J (2019) Repositioning salicylanilide anthelmintic drugs to treat adenovirus infections. Sci Rep 9(1):1

20. Moubarak M, Kasozi KI, Hetta HF, Shaheen HM, Rauf A, Alkuraishy $\mathrm{HM}$ et al (2021) The rise of SARS-CoV-2 variants and the role of convalescent plasma therapy for management of infections. Life 11(8):734

21. Al-Kuraishy HM, Al-Gareeb AI, Alblihed M, Guerreiro SG, CruzMartins N, Batiha GE (2021) COVID-19 in relation to hyperglycemia and diabetes mellitus. Front Cardiovasc Med 8:644095

22. Yuan J, Yun H, Lan W, Wang W, Sullivan SG, Jia S, Bittles AH (2006) A climatologic investigation of the SARS-CoV outbreak in Beijing, China. Am J Infect Control 34(4):234-236

23. Azhar EI, Hui DS, Memish ZA, Drosten C, Zumla A (2019) The middle east respiratory syndrome (MERS). Infect Dis Clin 33(4):891-905

24. Wu CJ, Jan JT, Chen CM, Hsieh HP, Hwang DR, Liu HW, Liu CY, Huang HW, Chen SC, Hong CF, Lin RK (2004) Inhibition of severe acute respiratory syndrome coronavirus replication by Niclosamide. Antimicrob Agents Chemother 48(7):2693-2696

25. Shie JJ, Fang JM, Kuo CJ, Kuo TH, Liang PH, Huang HJ, Yang WB, Lin CH, Chen JL, Wu YT, Wong CH (2005) Discovery of potent anilide inhibitors against the severe acute respiratory syndrome 3CL protease. J Med Chem 48(13):4469-4473

26. Gassen NC, Niemeyer D, Muth D, Corman VM, Martinelli S, Gassen A, Hafner K, Papies J, Mösbauer K, Zellner A, Zannas AS (2019) SKP2 attenuates autophagy through Beclin1-ubiquitination and its inhibition reduces MERS-Coronavirus infection. Nat Commun 10(1): $1-6$

27. Al-Kuraishy HM, Al-Gareeb AI, Qusty N, Cruz-Martins N, Batiha GE (2021) Sequential doxycycline and colchicine combination therapy in Covid-19: The salutary effects. Pulmonary Pharmacol Ther. 67:102008

28. Romani D, Noureddine O, Issaoui N, Brandán SA (2020) Properties and reactivities of Niclosamide in different media, a potential antiviral to treatment of COVID-19 by using DFT calculations and molecular docking. Biointerface Res Appl Chem 10:7295-7328

29. Guthappa R (2020) Molecular docking studies of N-acetyl cysteine, zinc acetyl cysteine and niclosamide on SARS Cov 2 protease and its comparison with hydroxychloroquine. Chemarxiv

30. Xu J, Shi PY, Li H, Zhou J (2020) Broad spectrum antiviral agent niclosamide and its therapeutic potential. ACS Infect Dis 6(5):909-915
31. Jeon S, Ko M, Lee J, Choi I, Byun SY, Park S, Shum D, Kim S (2020) Identification of antiviral drug candidates against SARSCoV-2 from FDA-approved drugs. Antimicrob Agents Chemother 64(7):e00819-e820

32. Brunaugh AD, Seo H, Warnken Z, Ding L, Seo SH, Smyth HD (2021) Development and evaluation of inhalable composite niclosamide-lysozyme particles: A broad-spectrum, patient-adaptable treatment for coronavirus infections and sequalae. PLOS ONE 16(2): 0246803

33. Wang G, Gaikwad H, McCarthy M, Gonzalez-Juarrero M, Li Y, Armstrong M, Reisdorph N, Morrison T, Simberg D (2021) Lipid nanoparticle formulation of niclosamide (nano NCM) effectively inhibits SARS-CoV-2 replication in vitro. Precis Nanomed 4(1):724-737

34. Jara MO, Warnken ZN, Sahakijpijarn S, Moon C, Maier EY, Christensen DJ, Koleng JJ, Peters JI, Hackman SD, Williams RO (2021) Niclosamide Inhalation powder made by thin-film freezing: pharmacokinetic and toxicology studies in rats and hamsters. bioRxiv

35. Liang L, Huang M, Xiao Y, Zen S, Lao M, Zou Y, Shi M, Yang X, $\mathrm{Xu} \mathrm{H}$ (2015) Inhibitory effects of Niclosamide on inflammation and migration of fibroblast-like synoviocytes from patients with rheumatoid arthritis. Inflamm Res 64(3):225-233

36. Cabrita I, Benedetto R, Schreiber R, Kunzelmann K (2019) Niclosamide repurposed for the treatment of inflammatory airway disease. JCI Insight 4(15):e128414

37. Costabile G, d'Angelo I, Rampioni G, Bondì R, Pompili B, Ascenzioni F, Mitidieri E, d'Emmanuele di Villa Bianca R, Sorrentino R, Miro A, Quaglia F (2015) Toward repositioning Niclosamide for antivirulence therapy of Pseudomonas aeruginosa lung infections: development of inhalable formulations through nanosuspension technology. Mol Pharm 12(8):2604-2617

38. Zhang LX, Zhao HJ, Sun DL, Gao SL, Zhang HM, Ding XG (2017) Niclosamide attenuates inflammatory cytokines via the autophagy pathway leading to improved outcomes in renal ischemia/reperfusion injury. Mol Med Rep 16(2):1810-1816

39. Hu B, Huang S, Yin L (2021) The cytokine storm and COVID-19. J Med Virol 93(1):250-256

40. Mehranfar A, Izadyar M (2020) Theoretical design of functionalized gold nanoparticles as antiviral agents against Severe Acute Respiratory Syndrome Coronavirus 2 (SARS-CoV-2). J Phys Chem Lett 11(24):10284-10289

41. Braga CL, Felix NS, Teixeira DE, Vieira JB, Silva-Aguiar RP, Bose RM, Antunes MA, de Novaes Rocha N, Caruso-Neves C, Cruz FF, Rocco PR (2020) Niclosamide attenuates lung vascular remodeling in experimental pulmonary arterial hypertension. Eur J Pharmacol 887:173438

42. Pagnesi M, Baldetti L, Beneduce A, Calvo F, Gramegna M, Pazzanese V, Ingallina G, Napolano A, Finazzi R, Ruggeri A, Ajello S (2020) Pulmonary hypertension and right ventricular involvement in hospitalised patients with COVID-19. Heart 106(17):1324-1331

43. Al-Kuraishy HM, Al-Gareeb AI, Faidah H, Al-Maiahy TJ, CruzMartins N, Batiha GE (2021) The looming effects of estrogen in Covid-19: a rocky rollout. Front Nutr 8:649128

44. Zuo Y, Warnock M, Harbaugh A, Yalavarthi S, Gockman K, Zuo M, Madison JA, Knight JS, Kanthi Y, Lawrence DA (2021) Plasma tissue plasminogen activator and plasminogen activator inhibitor-1 in hospitalized COVID-19 patients. Sci Rep 11(1):1-9

45. Kang S, Tanaka T, Inoue H, Ono C, Hashimoto S, Kioi Y, Matsumoto H, Matsuura H, Matsubara T, Shimizu K, Ogura H (2020) IL-6 trans-signaling induces plasminogen activator inhibitor-1 from vascular endothelial cells in cytokine release syndrome. Proc Natl Acad Sci USA 117(36):22351-22356

46. Wang LH, Xu M, Fu LQ, Chen XY, Yang F (2018) The antihelminthic Niclosamide inhibits cancer stemness, extracellular 
matrix remodeling, and metastasis through dysregulation of the nuclear $\beta$-catenin/c-Myc axis in OSCC. Sci Rep 8(1):1-3

47. Gyamfi J, Lee YH, Min BS, Choi J (2019) Niclosamide reverses adipocyte induced epithelial-mesenchymal transition in breast cancer cells via suppression of the interleukin-6/STAT3 signalling axis. Sci Rep 9(1):1-4

48. Al-Kuraishy HM, Al-Gareeb AI, Alzahrani KJ, Cruz-Martins N, Batiha GE (2021) The potential role of neopterin in Covid-19: a new perspective. Mol Cell Biochem 28:1-6

49. Gassen NC, Papies J, Bajaj T, Dethloff F, Emanuel J, Weckmann K, Heinz DE, Heinemann N, Lennarz M, Richter A, Niemeyer D (2020) Analysis of SARS-CoV-2-controlled autophagy reveals spermidine, MK-2206, and niclosamide as putative antiviral therapeutics. BioRxiv

50. He X, Li M, Ye W, Zhou W (2021) Discovery of degradable niclosamide derivatives able to specially inhibit small cell lung cancer (SCLC). Bioorg Chem 107:104574

51. Aloni-Grinstein R, Charni-Natan M, Solomon H, Rotter V (2018) p53 and the viral connection: back into the future. Cancers 10(6):178

52. Al-Kuraishy HM, Hussien NR, Al-Naimi MS, Al-Buhadily AK, Al-Gareeb AI, Lungnier C (2020) Is ivermectin-azithromycin combination the next step for COVID-19? Biomed Biotechnol Res J 4(5):101

53. Al-Kuraishy HM, Al-Gareeb AI, Alblihed M, Cruz-Martins N, Batiha GE (2021) COVID-19 and risk of acute ischemic stroke and acute lung injury in patients with type ii diabetes mellitus: the anti-inflammatory role of metformin. Front Med 8:110

54. Hu N, Fu Y, Li WF, Yang XR, Cao M, Li FF, Chen JH, Chen XY, Zhao H, Sun ZJ, Dong DL (2021) Chemical mitochondrial uncouplers share common inhibitory effect on NLRP3 inflammasome activation through inhibiting $\mathrm{NF} \times \mathrm{B}$ nuclear translocation. Toxicol Appl Pharmacol 21:5426

55. Ko M, Jeon S, Ryu WS, Kim S (2021) Comparative analysis of antiviral efficacy of FDA-approved drugs against SARS-CoV-2 in human lung cells. J Med Virol 93(3):1403-1408

56. Zhao J, He Q, Gong Z, Chen S, Cui L (2016) Niclosamide suppresses renal cell carcinoma by inhibiting $\mathrm{Wnt} / \beta$-catenin and inducing mitochondrial dysfunctions. Springerplus 5(1):1-8

57. Cecchini R, Cecchini AL (2020) SARS-CoV-2 infection pathogenesis is related to oxidative stress as a response to aggression. Med Hypotheses 143:102

58. Montopoli M, Zumerle S, Vettor R, Rugge M, Zorzi M, Catapano CV, Carbone GM, Cavalli A, Pagano F, Ragazzi E, Prayer-Galetti $\mathrm{T}$ (2020) Androgen-deprivation therapies for prostate cancer and risk of infection by SARS-CoV-2: a population-based study $(\mathrm{n}=$ 4532). Ann Oncol 31(8):1040-1045

59. Liu C, Armstrong C, Zhu Y, Lou W, Gao AC (2016) Niclosamide enhances abiraterone treatment via inhibition of androgen receptor variants in castration resistant prostate cancer. Oncotarget 7(22):32210

60. Wu CS, Li YR, Chen JJ, Chen YC, Chu CL, Pan IH, Wu YS, Lin CC (2014) Antihelminthic Niclosamide modulates dendritic cells activation and function. Cell Immunol 288(1-2):15-23

61. Sekulovski N, Whorton AE, Tanaka T, Hirota Y, Shi M, MacLean JA, de Mola JR, Groesch K, Diaz-Sylvester P, Wilson T, Hayashi K (2020) Niclosamide suppresses macrophage-induced inflammation in endometriosis. Biol Reprod 102(5):1011-1019

62. Ruscitti P, Bruno F, Berardicurti O, Acanfora C, Pavlych V, Palumbo P, Conforti A, Carubbi F, Di Cola I, Di Benedetto P, Cipriani P (2020) Lung involvement in macrophage activation syndrome and severe COVID-19: results from a cross-sectional study to assess clinical, laboratory and artificial intelligence-radiological differences. Ann Rheum Dis 79(9):1152-1155
63. Garrett T, Coatsworth H, Mahmud I, Hamerly T, Stephenson CJ, Yazd H, Ayers JB, Miller M, Lednicky JA, Dinglasan RR (2021) Niclosamide reverses SARS-CoV-2 control of lipophagy. bioRxiv

64. Centeio R, Cabrita I, Benedetto R, Talbi K, Ousingsawat J, Schreiber R, Sullivan JK, Kunzelmann K (2020) Pharmacological inhibition and activation of the $\mathrm{Ca} 2+$ activated $\mathrm{Cl}-$ channel TMEM16A. Int J Mol Sci 21(7):2557

65. Miner K, Labitzke K, Liu B, Wang P, Henckels K, Gaida K, Elliott R, Chen JJ, Liu L, Leith A, Trueblood E (2019) Drug repurposing: the anthelmintics niclosamide and nitazoxanide are potent TMEM16A antagonists that fully bronchodilate airways. Front Pharmacol 10:51

66. Li Z, Brecher M, Deng YQ, Zhang J, Sakamuru S, Liu B, Huang R, Koetzner CA, Allen CA, Jones SA, Chen H (2017) Existing drugs as broad-spectrum and potent inhibitors for Zika virus by targeting NS2B-NS3 interaction. Cell Res 27(8):1046-1064

67. Fan X, Xu J, Files M, Cirillo JD, Endsley JJ, Zhou J, Endsley MA (2019) Dual activity of niclosamide to suppress replication of integrated HIV-1 and Mycobacterium tuberculosis (Beijing). Tuberculosis 116:S28-33

68. Weiss A, Touret F, Baronti C, Gilles M, Hoen B, Nougairède A, de Lamballerie X, Sommer MO (2021) Niclosamide shows strong antiviral activity in a human airway model of SARS-CoV-2 infection and a conserved potency against the UK B. 1.1. 7 and SA B. 1.351 variant. bioRxiv

69. Jo S, Kim S, Shin DH, Kim MS (2020) Inhibition of SARSCoV 3CL protease by flavonoids. J Enzyme Inhib Med Chem 35(1): 145-151

70. Johnson TO, Adegboyega AE, Iwaloye O, Eseola OA, Plass W, Afolabi B, Rotimi D, Ahmed EI, Albrakati A, Batiha GE, Adeyemi OS (2021) Computational study of the therapeutic potentials of a new series of imidazole derivatives against SARS-CoV-2. J Pharmacol Sci

71. Chen L, Wang L, Shen H, Lin H, Li D (2017) Anthelminthic drug niclosamide sensitizes the responsiveness of cervical cancer cells to paclitaxel via oxidative stress-mediated mTOR inhibition. Biochem Biophys Res Commun 484(2):416-421

72. Niyomdecha N, Suptawiwat O, Boonarkart C, Jitobaom K, Auewarakul $P$ (2020) Inhibition of human immunodeficiency virus type 1 by niclosamide through mTORC1 inhibition. Heliyon 6(6):e04050

73. Bonaventura A, Vecchié A, Dagna L, Martinod K, Dixon DL, Van Tassell BW, Dentali F, Montecucco F, Massberg S, Levi M, Abbate A (2021) Endothelial dysfunction and immunothrombosis as key pathogenic mechanisms in COVID-19. Nat Rev Immunol 21(5):319-329

74. Engin S, Yasar YK, Barut EN, Sezen SF (2021) Improved endothelium-dependent relaxation of thoracic aorta in niclosamide-treated diabetic rats. Cardiovasc Toxicol 27:1-9

75. Laubscher GJ, Lourens PJ, Venter C, Grobbelaar LM, Kell DB, Pretorius E (2021) A decision-tree approach to treat platelet hyperactivity and anomalous blood clotting in acute COVID-19 patients. medRxiv

76. Hamilton G, Rath B (2017) Repurposing of anthelminthics as anticancer drugs. Oncomedicine 2:142-149

77. Ke R, Xu Q, Li C, Luo L, Huang D (2018) Mechanisms of AMPK in the maintenance of ATP balance during energy metabolism. Cell Biol Int 42(4):384-392

Publisher's Note Springer Nature remains neutral with regard to jurisdictional claims in published maps and institutional affiliations. 